\title{
Reconstruction and Analysis of Pulsed Thermographic Data
}

Steven M. Shepard, James R. Lhota, Yulin Hou, David Wang and Tasdiq Ahmed Thermal Wave Imaging, Inc. 845 Livernois, Ferndale, MI 48220, USA sshepard@thermalwave.com

KEYWORDS: nondestructive, infrared, thermography

\section{ABSTRACT}

Interpretation of pulsed thermographic data sequences has typically been based on contrast analysis, in which regions with subsurface anomalies are visually identified with respect to defect-free areas. Quantitative contrastbased analysis requires that the time history of a defect-free reference point, either real or synthetic, is subtracted from the time history of a given point in order to create a contrast curve. In practice, this approach has been limited by the fact that real samples may be either completely defective or intact, so that no contrast exists within the field of view. Although numerous variants to this approach have been developed, they all require the identification of a defect-free region as a reference.

Thermographic Signal Reconstruction (TSR) is a viable alternative to contrast analysis, as it allows non-visual interrogation of thermographic data sequences. The patented TSR technique recognizes the nearly linear behavior of the logarithmic representation of a pixel's time history, and exploits this characteristic by representing the logarithmic data sequence as a series of functions, e.g. a low order polynomial, obtained through a least squares fit to the data. Once the series representation has been created, a replica the original data set can be reconstructed, identical in every respect except the presence of temporal noise. For a given image in the sequence, it is difficult to discriminate between the original and reconstructed images, as the only difference is the high spatial frequency "salt and pepper " noise in the original image. Thus, the reconstructed image offers improved signal to noise performance, but minimal improvement to the contrast to noise ratio.

The more significant aspect of the reconstruction process is the ability to operate on the series representation of the pixel time history. For example, 
first or second time derivatives of any image in the data sequence can be created nearly instantaneously. The derivative images offer an extremely clear visualization of subsurface features, and in many cases, it is possible to detect features in derivative images that are totally undetectable in normal contrast images. The derivative images offer a high degree of immunity to nonuniform heating, and display less blurring than contrast images, as features may be detected earlier, before significant lateral diffusion occurs. Non-visual interpretation of the reconstructed sequences is readily performed by identifying inflection points in the series. To archive the reconstructed data, only the coefficients of the series are stored, so that a significant degree of data compression occurs. As a result, it is possible to process and analyze multiple sequences simultaneously. For a 400 -frame sequence of $320 \times 256$ pixel, 14-bit data, conversion time is typically less than 10 seconds, and file size is reduced from $46 \mathrm{MB}$ to $4.8 \mathrm{MB}$. 